\title{
L'ORFEO TOOLBOX : BILAN DES ANNÉES ORFEO ET PERSPECTIVES
}

\author{
Julien Michel ${ }^{1}$, Manuel Grizonnet ${ }^{1}$, Jordi Inglada ${ }^{2}$ \\ 1: CNES, 18 avenue Edouard Belin, BPI 1219, 31401 TOULOUSE CEDEX 9 \\ 2: CNES-CESBIO, 18 avenue Edouard Belin, BPI 2801, 31401 TOULOUSE CEDEX 9
}

\begin{abstract}
Résumé
Dans le cadre du programme d'accompagnement ORFEO de la mission Pléiades, le CNES développe depuis 2006 l'Orfeo ToolBox, logiciel libre de traitement d'image de télédétection. L'objectif du logiciel est double : permettre une capitalisation du savoir faire méthodologique développé pour ORFEO, et mettre à la disposition des utilisateurs des outils génériques facilitant l'exploitation des données Pléiades par les utilisateurs thématiques. Après 8 années de développement et presque 3 années de vie du système Pléiades en vol, cet article mesure le chemin parcouru et dessine le futur de l'Orfeo ToolBox.
\end{abstract}

Mots clés : Orfeo ToolBox, Logiciel Libre, Télédétection

\begin{abstract}
In the frame of the ORFEO accompaniment program to Pléiades, CNES develops since 2006 the Orfeo ToolBox, a free and open source software dedicated to image processing for remote sensing. The main objectives of the software are twofold : capitalization of the methodological knowledge built during ORFEO, and availability of generic tools to work with Pléiades data for ORFEO thematic users. After 8 years of development and almost 3 years of in-flight life of Pléiades, this article sums up the progress made and what the future of OTB might be.
\end{abstract}

Keywords : Orfeo ToolBox, Open Source Software, Remote Sensing

\section{Introduction}

Dans le cadre du volet méthodologique du programme d'accompagnement ORFEO préparant et promouvant l'utilisation des données issues des systèmes Pléiades (PHR) et Cosmo-Skymed (CSK), le Centre National d'Études Spatiales (CNES) a décidé en 2006 de développer l' ORFEO Toolbox (OTB), un ensemble de briques algorithmiques qui permettent de capitaliser le savoir méthodologique et de se placer dans une démarche de développement incrémental.

L'ORFEO Toolbox (OTB) est distribuée sous la forme d'une librairie open source de traitement d'image sous licence CeCILL-V2 ${ }^{1}$. Elle est construite à partir de la librairie de traitement d'image ITK et propose des fonctionnalités adaptées à la télédétection et particulièrement aux images à très haute résolution spatiale.

L'OTB propose un grand nombre de fonctions documentées et se décline dans différents outils comme par exemple le logiciel Monteverdi qui permet la visualisation et le traitement des données Pléiades via une interface pratique.

En huit ans, l'OTB a fédéré une large communauté d'utilisateurs d'images de télédétection, et ce succès en fait l'un des outils de référence pour l'exploitation et le traitement d'images optiques très haute résolution telles que celles fournies par Pléiades.

1. http://cecill.info/licences/Licence_ CeCILL_V2-fr.html

\subsection{Pourquoi l'Orfeo ToolBox?}

Les images de télédétection sont très riches en informations, cependant l'extraction automatique de ces informations est souvent un processus complexe, et l'interprétation visuelle des images reste bien souvent la méthode la plus fiable. Le support des formats, et les prétraitements nécessaires pour générer les données sont des tâches qui peuvent avoir un impact majeur sur la performance des algorithmes.

Concernant ces algorithmes d'extraction, un problème récurrent est l'écart entre des méthodes récentes issues de l'état de l'art, très prometteuses mais qui nécessitent souvent un paramétrage fin et une bonne compréhension de la théorie, et les méthodes utilisées en production. De plus, ces méthodes récentes manquent souvent d'une mise en œuvre de référence les rendant accessibles à un utilisateur.

L'idée de l'OTB est de réduire cet écart en fournissant aux utilisateurs un panel complet d'outils et d'algorithmes inter-opérables permettant de faciliter l'analyse et l'extraction d'information utile dans les images optiques Très Haute Résolution telles que celles fournies par Pléiades. De plus, l'OTB se veut être un réceptacle pour collecter sous une interface commune et diffuser différents résultats issues de la R\&D du CNES en extraction d'information (Inglada et Christophe, 2009; Christophe et al., 2011).

En plus de la mise à disposition de ces briques algorithmiques, l'objectif de l'OTB est de fournir une interface générique permettant de mettre en œuvre ces traitements à grande échelle et de manière efficace en paral- 


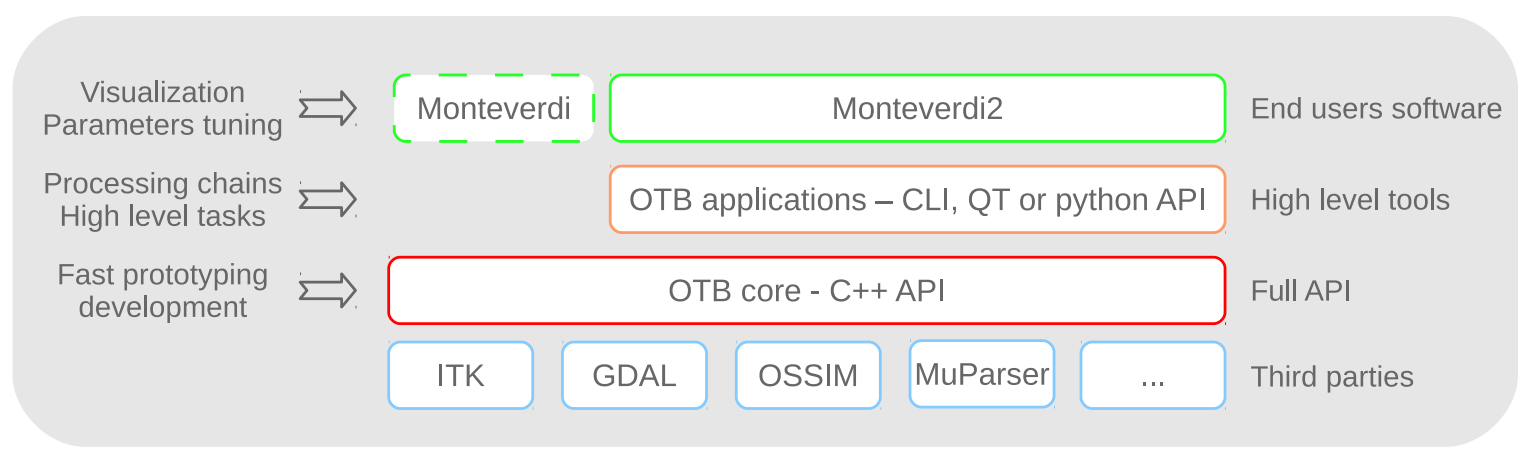

Figure 1: Architecture et moyens d'accès de l'Orfeo ToolBox

lélisant les opérations. Ceci est rendu nécessaire par le volume des images optiques THR et est un élément différenciant par rapport à d'autres solutions de traitement d'image de télédétection (Christophe et al., 2011).

Cette librairie étant destinée aux utilisateurs du programme ORFEO et au delà aux utilisateurs de l'imagerie spatiale, sa gratuité et sa publicité se comprennent aisément. Cependant, le CNES a souhaité aller au delà en la diffusant sous la forme d'un logiciel libre, dont les sources ainsi que l'ensemble des ressources de développement sont publiques et librement accessibles. Les motivations de ce caractère open-source sont multiples :

- Adopter une démarche de recherche reproductible : le libre accès au code source facilite en effet l'évaluation, la validation et la comparaison et l'amélioration des méthodes développées par la communauté scientifique,

- Fiabiliser les mises en œuvre en permettant à chacun de rechercher l'origine d'anomalies ou d'en comprendre le comportement en lisant le code source,

- Favoriser les contributions de la communauté méthodologique et la réutilisation du logiciel dans des projets tiers sur le principe du bénéfice mutuel,

- Favoriser les échanges avec les logiciels tiers (tous open-source) et s'intégrer dans le microcosme des logiciels libres d'information géographique.

- Faire de l'OTB une vitrine du savoir faire collecté autour de l'imagerie Pléiades mais aussi de l'imagerie optique Très Haute Résolution en général.

\subsection{Qu'est l'Orfeo ToolBox?}

L'Orfeo ToolBox est structurée autour de différents éléments qui sont résumés dans le schéma proposé figure 1.

Tout d'abord, la librairie s'appuie sur un ensemble de logiciels tiers dont les principaux sont ITK ${ }^{2}$ pour l'architecture logicielle globale ainsi que les fonctionnalités de traitement d'image qui y sont disponibles, $\mathrm{GDAL}^{3}$ pour l'accès en lecture et écriture des données géo-spatiales, et $\mathrm{OSSIM}^{4}$ pour la gestion des modèles géométriques

\footnotetext{
2. wWw.itk. org

3. www. gdal.org

4. www. ossim.org
}

et des projections cartographiques. Le coeur de l'OTB consiste en une extension d'ITK écrite en $\mathrm{C}++$ et offrant des fonctionnalités de traitement d'images de télédétection s'appuyant sur ces logiciels tiers et des développements propres de certains algorithmes. Cette API C++ très riche et modulaire peut être utilisée pour construire rapidement des chaînes de traitement qui de par la mécanique mise oeuvre par ITK, tirera avantage d'une architecture de calcul multi-cœur et traitera séquentiellement les gros volumes de données afin de ne pas saturer la mémoire.

Aux utilisateurs qui ne souhaitent pas développer leurs propres chaînes de traitement en $\mathrm{C}++_{+}$, l'OTB propose une suite d'outils appelables en ligne de commande, via une interface graphique en QT ou au travers d'une API Python de haut-niveau. Ces outils réalisent des tâches courantes en traitement d'image et en télédétection et s'appuient sur l'API C++ sous-jacente de l'OTB. Ces outils permettent de capitaliser le savoir-faire (opérations à enchaîner, paramétrage, documentation ...) nécessaire à une fonction donnée en un unique point d'accès facilement utilisable par l'utilisateur. Des exemples de fonctions de haut-niveau sont l'ortho-rectification, la classification ou la segmentation. L'OTB intègre actuellement près de 74 applications différentes, et cet ensemble peut être étendu par des applications développées par l'utilisateur lui-même par le biais d'un mécanisme de greffons.

Enfin, l'Orfeo ToolBox propose au travers des logiciels Monteverdi d'abord, puis Monteverdi2 ensuite, un logiciel intégré permettant à l'utilisateur final à la fois la visualisation d'image et le traitement interactif de ces images. Ces outils se sont révélés très utiles pour l'animation de formations et sont également une porte d'entrée simple et accessible vers les modes d'accès plus complexes décrits ci-dessus.

II faut également noter l'objectif capital de disponibilité de l'outil sur l'ensemble des systèmes d'exploitation courants (Linux, Windows, Mac OSX), qui a été et reste une contrainte forte de développement de l'Orfeo ToolBox, mais également une réelle force du projet.

II est difficile de dresser ici une liste exhaustive des fonctionnalités disponibles, cependant les fonctionnalités principales offertes par la librairie sont les suivantes:

- accès à l'image : accès optimisé en lecture et écri- 
ture pour la plupart des formats d'image de télédétection et à leurs métadonnées au travers des librairies Gdal et OSSIM, support des formats SIG vecteurs,

- Traitements géométriques : localisation directe et inverse par modélisation du capteur, projections cartographiques, superposition d'images, ortho-rectification, stéréo-rectification,

- Traitements radiométriques : corrections atmosphériques, indices radiométriques,

- Filtrages linéaires et non-linéaires usuels, réduction de la dimension,

- Fusion : pansharpening P+XS, fusion de cartes de classifications,

- Extraction de primitives : points d'intérêt (SIFT, SURF), lignes (LSD), textures, histogrammes de gradients orientés, etc ...

- Segmentation d'images : croissance de régions, Mean-Shift, ligne de partage des eaux, profils morphologiques,

- Classification supervisée (SVM, Random Forest ... ) et non supervisée (K-means, champs de Markov, cartes de Kohonen),

- détection des changements : opérateurs usuels, Multivariate Alteration Detector

- Analyse d'image orientée objet : conversion raster vers vecteur, filtrage par attributs,

- Traitement d'images hyperspectrales : estimation de la dimensionnalité, extraction d'endmembers, démélangeage.

\section{L'Orfeo ToolBox de 2006 à 2014}

La figure 2 montre la chronologie des différentes versions de l'Orfeo ToolBox, ainsi que l'évolution de certains chiffres clés sur la période 2006-2014.

\subsection{La version $\mathbf{1 . 0}$}

Les premières versions ont essentiellement été dédiées à poser les bases du logiciel. Outre l'architecture générale conçue comme une extension de la librairie ITK, le support des formats des images de télédétection a été réalisé par le biais de la librairie Gdal. Ces versions ont également permis de réaliser les premiers développements d'algorithmes, démontrant ainsi l'adéquation du design ITK avec les besoins. Enfin, c'est durant cette période que les principes de génie logiciel utilisés ont été mis en place : développement guidé par les tests, système de validation quotidienne automatisé, documentation, développement agile. A ce stade, la librairie se compose exclusivement de l'API C++ ainsi que de petits programmes d'exemples documentés dans le guide utilisateur (OTB development team, 2014b).

\subsection{Entre les versions 1.0 à 2.0}

L'Orfeo ToolBox a ensuite connu une seconde phase durant laquelle le principal objectif a été l'enrichissement des fonctionnalités disponibles. De nombreux algorithmes

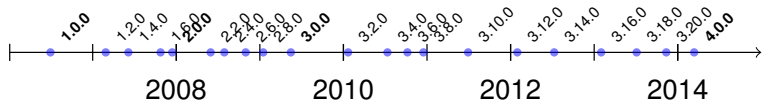

(1) Chronologie des versions de l'Orfeo ToolBox

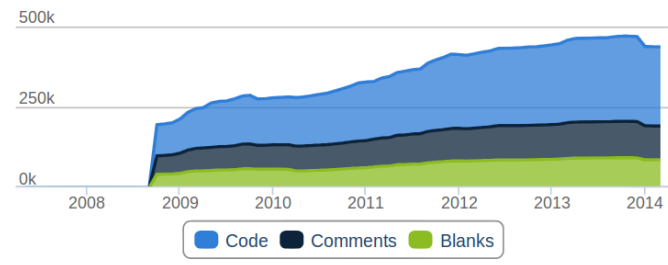

(2) Évolution du volume de code (en nombre de lignes, source : ohloh.net)

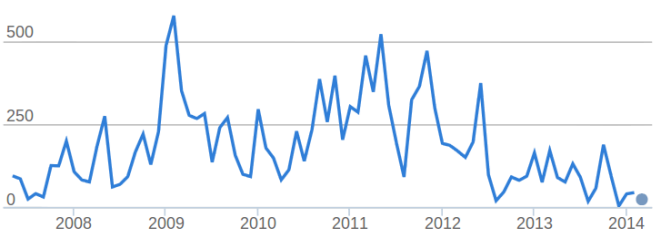

(3) Évolution de l'activité de développement (en commits par mois, source : ohloh.net)

Figure 2: Évolutions de l'Orfeo ToolBox entre 2006 et 2014

ont ainsi été rajoutés pour l'extraction de primitives (textures, extraction de contours ...), la segmentation (MeanShift), la classification des images (algorithme SVM). Le moyen d'accès privilégié reste l'API $\mathrm{C}++_{+}$et le guide utilisateur.

\subsection{De la version 2.0 à la version 3.2 .0}

En parallèle de la mise à disposition de nouveaux algorithmes de traitement d'images, les utilisateurs non développeurs ont exprimé le besoin d'avoir accès à ces traitements via des interfaces graphiques permettant de les tester et de les paramétrer plus rapidement.

Des applications de démonstration avec une interface graphique ont donc été ajoutées à l'écosystème OTB, dessinant ainsi la voie vers un logiciel graphique complet intégré. En 2009, l'opportunité de développer un tel logiciel est fournie par la Direction de la Stratégie et des Programmes du CNES, qui en finance le développement pour des besoins de formations dispensées par le CNES dans les pays émergents. Le succès de cet applicatif appelé Monteverdi, qui sort en même temps que la version 3.2.0, dépasse de loin ce cadre initial. Par son biais, de nombreux utilisateurs découvrent les fonctionnalités et commencent à les utiliser pour leurs travaux au quotidien. Parallèlement, l'enrichissement des fonctionnalités continue, avec notamment des travaux autour de I'OBIA (Object Based Image Analysis).

Durant cette période, on constate également l'essor d'une communauté OTB qui échange et participe à la vie du projet via un ensemble de médias (liste de diffusion, forum, blogs, réseaux sociaux...). 


\subsection{De la version 3.2 .0 à la version 4.0 .0}

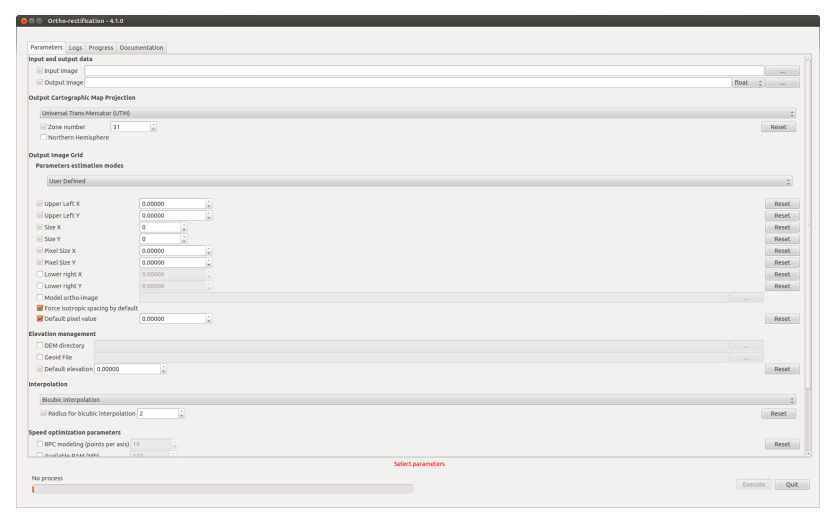

FIGURE 3: Un exemple d'interface graphique QT autogénérée pour une application

A partir de la version 3.12.0, un nouveau pas est franchi dans les moyens d'accès à l'OTB, avec le développement du framework des applications. Celui-ci part d'un constat simple : entre Monteverdi et les applications de démonstration, de nombreuses chaînes de traitement réalisant des tâches courantes (ortho-rectification, classification ...) sont écrites plusieurs fois dans des versions qui diffèrent légèrement, ce qui augmente inutilement le coût de maintenance et favorise des comportements incohérents d'une chaîne à l'autre.

Pour pallier à ce problème, un modèle d'application autodescriptive et auto-documentée est développé. Celuici permet d'implémenter une chaîne de traitement et de décrire et documenter ses paramètres, de sorte qu'elle soit appelable au travers de différentes interfaces : $\mathrm{C}_{++}$, Python, ligne de commande, interface auto-générée en QT (voir figure 3) ... L'idée est de disposer d'une unique mise en œuvre de chaque chaîne de traitement, qui puisse ensuite être ré-utilisée dans tout les contextes, et interfacée avec les logiciels qui le souhaitent. De plus, cette architecture se base sur un système de greffons qui permet à l'utilisateur d'enrichir les applications disponibles. Une fois en place, ce mode d'accès est plébiscité par les utilisateurs, principalement pour sa simplicité et sa grande versatilité. II permet également à l'Orfeo ToolBox de distribuer des fonctions de haut-niveau s'appuyant sur la librairie. Parallèlement à l'essor de ces applications, un guide d'utilisateur pour les non-développeurs, qui contient la documentation de référence des applications ainsi que des tutoriaux pour les tâches courantes de traitement d'image, est réalisé (OTB development team, 2014a).

II faut également noter que la version 3.12.0, synchrone du tir de Pléiades $1 \mathrm{~A}$, propose déjà un support complet de l'imagerie Pléiades et de ses métadonnées.

Suite au développement des applications OTB, il a été décidé de refondre complètement le logiciel Monteverdi. La nouvelle mouture, dont la première version coïncide avec la version 3.16.0 de l'Orfeo ToolBox, propose entre autre une interface plus moderne en $\mathrm{QT}$, une navigation dans les images plus moderne également, un

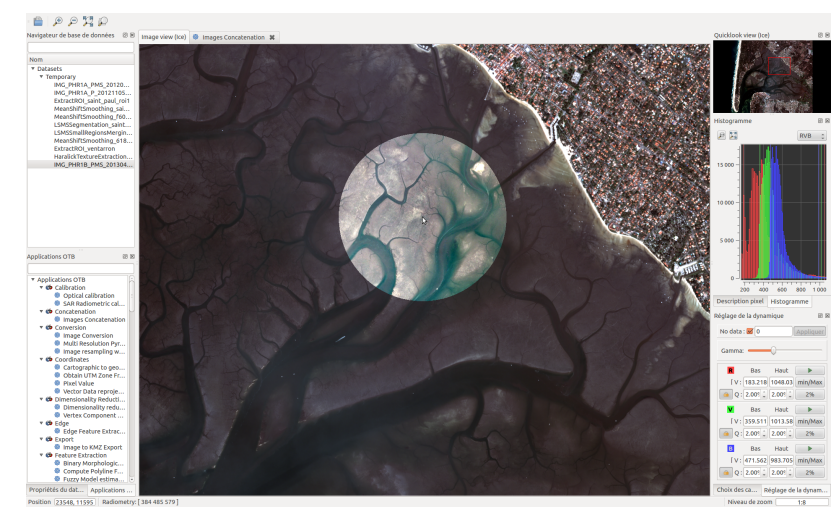

FIGURE 4: L'interface graphique de Monteverdi2

système de gestion des données persistant, à l'instar des logiciels de gestion de photos, et une internationalisation native. L'interface graphique de ce logiciel, appelé Monteverdi2, est présentée figure 4.

\subsection{L’Orfeo ToolBox 4.0 : Préparer le futur}

Comme expliqué section 1.2, l'OTB est basée sur de nombreuses bibliothèques open-source. En particulier, le cœur du système OTB s'appuie sur la librairie de traitement d'images médicales Insight Toolkit (ITK), qui a démarré en 2011 la transition vers une nouvelle version majeure appelée la série 4.0 (ITKv4). Le projet a décidé de remettre à plat un certain nombre de choix de design et d'interface, ce qui s'est traduit par des changements d'API majeurs. Mais cette version apporte également un grand nombre de fonctionnalités et vise à faciliter encore le développement de chaînes de traitement d'images. Durant toute l'année 2013, l'équipe de développement de l'OTB a travaillé pour rendre compatible l'OTB avec cette nouvelle version. Cet effort s'est accompagné, tout comme l'a été le développement de la version 4.0 d'ITK, de la mise en place d'une documentation appropriée permettant de faciliter pour les utilisateurs de la librairie la migration vers cette nouvelle version. Le résultat de ce travail est le sortie de la version 4.0.0 de l'Orfeo ToolBox, compatible de la version 4.0.0 d'ITK, qui pose des bases solides pour la suite des développements.

\section{Chiffres clés, succès, axes d'amélioration}

\subsection{Quelques chiffres clés}

Combien d'utilisateurs compte l'Orfeo ToolBox? Du fait du caractère libre du logiciel et de sa facilité d'accès, répondre à cette question est difficile. Néanmoins, on peut noter que la liste de diffusion dédiée aux utilisateurs compte 577 membres et que 100 à 150 messages sont échangés tous les mois entre les utilisateurs par ce biais. La liste réservée aux développeurs, également publique, compte 89 membres. II y a également 
118 comptes enregistrés dans le système de déclaration et de suivi des anomalies en ligne, et 52 contributeurs à la rédaction du Software Guide (OTB development team, 2014b). Le site de distribution de logiciels libres sourceforge.net utilisé pour diffuser les sources de l'Orfeo ToolBox recense 5742 téléchargements depuis la date de sortie de la version 4.0.0. La figure 5 montre les statistiques de téléchargements fournies par le site sourceforge.net distribuant les sources de l'Orfeo ToolBox.

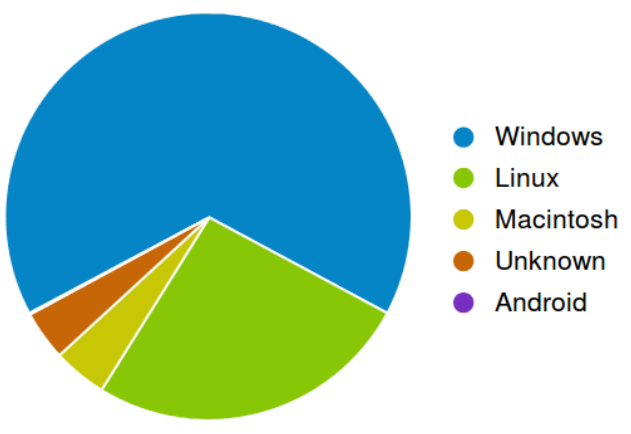

(1) Téléchargements par système d'exploitation

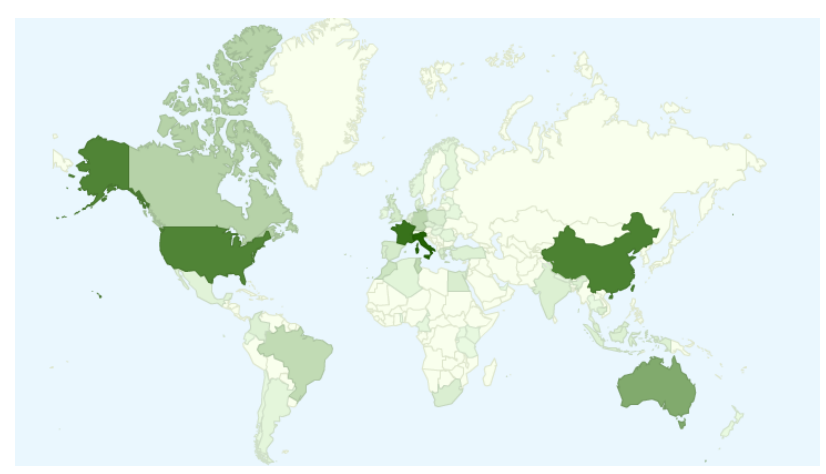

(2) Téléchargements par pays

FIGURE 5: Statistiques de téléchargement (source : sourceforge.net)

\subsection{Les succès de l'OTB}

L'Orfeo ToolBox a été un outil de support important pour les phases préparatoires et Recette Thématique Utilisateurs Pléiades. En plus d'une utilisation directe par certains acteurs de la RTU comme le CEREMA, ou le laboratoire EGID par exemple, l'Orfeo ToolBox a été l'outil principal de l'équipe de support méthodologique du CNES durant toute la phase de recette thématique. Ainsi, de nombreuses tâches de pré-traitement (otho-rectification sur SRTM, fusion $\mathrm{P}_{+} \mathrm{XS}$ ), mais également de traitement (segmentation) ont été réalisées à l'aide de l'outil. Par ailleurs, au sein de la plateforme de traitement aval mise en place par le CNES dans le cadre de la RTU, I'OTB a traité avec succès plus de 619 images Pléiades entières, à la fois pour des tâches de pré-traitement et d'extraction d'information. Enfin, il faut mentionner que l'OTB a été le premier logiciel libre compatible avec le format des données Pléiades (format JPEG2000 et lecture des métadonnées). Cette compatibilité a par ailleurs été reversée aux logiciels libres tiers OpenJpeg v2 (décodage des fichiers JPEG2000), Gdal (intégration d'un driver OpenJpeg v2) et OSSIM support des métadonnées et du modèle de capteur, de sorte que l'ensemble de l'écosysème des logiciels SIG libre a bénéficié des avancées réalisées dans le cadre du développement de l'OTB.

Certaines fonctions proposées par l'OTB égalent ou dépassent celles proposées par les logiciels open-source et commerciaux du domaine, par exemple :

- La calculatrice raster est extrêmement riche et flexible par rapport à la concurrence grâce à l'utilisation de la librairie MuParser,

- La segmentation de scènes complètes sans artefacts de tuilage, et avec une garantie théorique d'un résultat exact par rapport à celui qu'aurait produit la même segmentation sans tuilage (Michel et al., 2014),

- La classification supervisée de scènes entières avec un grand choix d'algorithmes d'apprentissage,

- Les fonctionnalités facilitant l'intégration de données extraites des images de télédétection dans des logiciels SIG.

De plus, l'Orfeo ToolBox est utilisée dans plusieurs projets logiciels tels que :

- Gnorasi, logiciel développé par la National Technical University of Athens, qui permet la programmation visuelle d'une chaîne de traitement d'image,

- Le projet Vahine de I'IPAG, un outil pour le traitement des données astrophysiques hyperspectrales,

- Le projet SEAS de l'IRD pour le traitement d'images SPOT en pied d'antenne,

- Certains composant des segments sols Sentinel2 et Venus sont réalisés à base d'OTB,

- Le programme de recherche TCM de l'Ecole de Technologie Supérieure du Québec,

- Le programme de recherche FP7 Tolomeo,

- Le module de traitement de donnée de QGIS qui donne accès à l'ensemble des applications disponibles dans l'OTB. Ce module a par exemple été utilisé pour une formation réalisée par IGN France International et ONF International au Gabon (Lardeux, 2014).

\subsection{Les axes d'amélioration}

Les succès nombreux de l'Orfeo ToolBox dans le cadre ou en dehors du programme préparatoire ORFEO ne doivent pas faire oublier les difficultés et axes d'améliorations possibles, déclinés ici du point de vue de l'utilisateur et de celui du développeur.

En ce qui concerne l'utilisateur, l'un des freins principaux à l'utilisation de l'Orfeo ToolBox a été et est toujours sa courbe d'apprentissage. L'absence d'interface graphique ou même d'outil en ligne de commande a longtemps réduit l'audience de l'OTB aux utilisateurs familiers du langage $\mathrm{C}_{++}$, et cette image de logiciel difficile à utiliser a perduré même après la mise en place de moyens adaptés à l'utilisateur non développeur, tels que les applications ou encore les logiciels Monteverdi et Monteverdi2. Encore aujourd'hui, les utilisateurs les plus aven- 
tureux se heurtent souvent aux difficultés liées à la compilation de l'outil ou se perdent dans une documentation riche mais foisonnante.

Du point de vue du développeur, le $\mathrm{C}++$ et surtout la conception mélangeant polymorphisme et généricité, tout en devant se conformer aux concepts de la librairie ITK, aboutissent parfois à de véritables empilements de classes même pour réaliser des tâches très simples. De plus, la réutilisation intensive de logiciels libres tiers, si elle permet de proposer très rapidement de grandes familles de fonctions validées par la communauté pour un coût de développement inférieur (par rapport au développement complet de ces fonctions), possède ses propres contreparties. Ainsi il faut se conformer aux évolutions des différentes API, gérer la présence d'anomalies (en plus de celles de l'OTB), et composer avec les orientations des différents logiciels, qui ne sont pas toujours compatibles de celles souhaitées par l'équipe de développement pour l'OTB. Tout ceci se fait au travers de beaucoup d'échanges avec les différentes communautés, qui sont très riches et permettent de faire progresser globalement la qualité de l'ensemble des logiciels concernés, mais avec un coût important en terme de développement. A titre d'exemple, le passage de l'OTB à la version 4.0.0 d'ITK représente plus d'une année de travail.

Enfin, il faut rappeler que le succès des logiciels libres dépend souvent de leur adoption par la communauté des utilisateurs. En s'entre-aidant, en testant et validant les fonctionnalités, en reportant des anomalies, les utilisateurs contribuent à l'amélioration du logiciel et à son succès. La construction d'une telle communauté est un travail à long terme, qui commence à porter ses fruits pour l'Orfeo ToolBox : de nombreux utilisateurs cherchent à contribuer au projet en reportant systématiquement les anomalies rencontrées ou en répondant aux questions des autres utilisateurs sur la liste de diffusion par exemple. Cet investissement de l'utilisateur dans la vie du projet est une des clés du succès pour un logiciel, car il est impossible de contrôler et maîtriser au niveau de l'équipe de développement la variété des cas d'utilisation et d'environnement d'exécution.

\section{Conclusions et perspectives}

La rétrospective de ces huit années de développement permet de mesurer le chemin parcouru par l'Orfeo ToolBox, passant du status de prototype $\mathrm{C}++$ confidentiel à celui d'un logiciel largement reconnu et utilisé dans la communauté scientifique de la télédétection. Ce succès revient avant tout aux différents acteurs et contributeurs du projet, qui ont su réaliser cette transformation en y mettant leur passion de la télédétection, du développement logiciel et du logiciel libre.

Le terme du programme d'accompagnement ORFEO marque la fin d'une période pour l'Orfeo Toolbox, mais le CNES s'est déjà engagé à continuer les développements de l'outil, qui a par ailleurs déjà fait ses preuves en dehors du cadre ORFEO. L'objectif de cette nouvelle phase de développement est d'assurer la continuité du service pour les utilisateurs de la donnée Très Haute Résolution, mais aussi de s'ouvrir à d'autres capteurs et missions (SWOT, Sentinel 1 et 2, OTOS ...), et aux nouveaux besoins remontés par les utilisateurs de la librairie.

Le projet souhaite également confirmer sa position dans la communauté du logiciel libre pour les Systèmes d'Information Géographique, avec notamment le processus d'incubation pour entrer dans les projets soutenus par la fondation OSGeo ${ }^{5}$. Afin de renforcer encore le statut de logiciel libre, le CNES souhaite également mettre en place une gouvernance officielle par le biais d'un comité de pilotage ouvert aux participants extérieurs, dont la sélection se fera sur la base de leur investissement dans le projet.

Du coté technique, le support de ITK 4.0 a été un grand pas en avant, et ouvre la voie à plus de rationnalisation et de réduction du volume de code source. A l'instar d'ITK, nous souhaitons également pouvoir modulariser l'Orfeo ToolBox afin de faciliter encore son utilisation et d'encourager les contributions en limitant les impacts qu'elles ont sur l'ensemble du code source. Enfin, de nombreuses fonctionnalités sont déjà identifiées pour les années à venir, avec notamment plus d'OBIA, d'extraction de primitives, et de machine learning!

\section{Références}

Christophe, E., Michel, J., Inglada, J., 2011. Remote sensing processing : From multicore to gpu. Selected Topics in Applied Earth Observations and Remote Sensing, IEEE Journal of 4 (3), 643-652.

Inglada, J., Christophe, E., 2009. The orfeo toolbox remote sensing image processing software. Dans : IGARSS (4). pp. 733-736.

Lardeux, C. e. A. H., 2014. Qgis et ses outils de traitement pour l'analyse de l'occupation des terres. Dans : FOSS4G-FR.

Michel, J., Youssefi, D., Grizonnet, M., 2014. Stable mean-shift algorithm and its application to the segmentation of arbitrarily large remote sensing images. Geoscience and Remote Sensing, IEEE Transactions on PP (99), 1-13.

OTB development team, 2014a. The Orfeo ToolBox Cookbook, a guide for non-developers Updated for OTB-4.0.0. Cnes.

OTB development team, 2014b. The Orfeo ToolBox Software Guide, Updated for OTB-4.0.0. Cnes.

\footnotetext{
5. www. osgeo.org
} 